\title{
Surveillance de la résistance aux antimicrobiens dans les hôpitaux canadiens, 2007-2012
}

\author{
Gravel $D^{1^{*}}$, Archibald $C P^{1}$, Pelude $L^{1}$, Mulvey $M^{2}$ et Golding $G^{2}$ \\ au nom du Programme canadien de surveillance des infections nosocomiales
}

\footnotetext{
${ }^{1}$ Centre de la lutte contre les maladies transmissibles et les infections, Agence de la santé publique du Canada, Ottawa (Ontario)

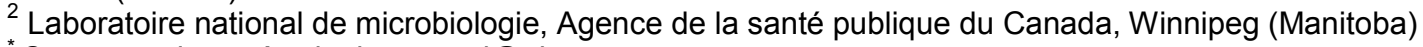

Correspondance à : denise.gravel@phac-aspc.gc.ca
}

\section{Résumé d'article}

Contexte : Le Programme canadien de surveillance des infections nosocomiales (PCSIN) est un effort concerté du Centre de lutte contre les maladies transmissibles et les infections de l'Agence de la santé publique du Canada, du Laboratoire national de microbiologie et de 54 grands hôpitaux sentinelles de soins tertiaires affiliés à une université dans 10 provinces du Canada.

Objectif : Fournir un résumé des taux de résistance aux antibiotiques de quatre organismes clés résistant aux antibiotiques dans les hôpitaux majeurs du pays entre le $1^{\text {er }}$ janvier 2007 et le 31 décembre 2012.

Méthodologie : Les données cliniques et démographiques des patients ainsi que les résultats associés des analyses de laboratoire ont été soumis à l'Agence par les hôpitaux participants. Les taux d'infection ont été résumés par 1000 hospitalisations aux niveaux national et régional.

Résultats : Au Canada, les taux globaux d'infections à Clostridium difficile (ICD) associées aux soins de santé ont atteint un sommet en 2008 (5,8 ICD associées aux soins de santé pour 1000 hospitalisations), puis sont demeurés stables entre 2009 et 2012 (environ 5 ICD associées aux soins de santé pour 1000 hospitalisations), les régions de l'Ouest et du Centre du Canada présentant des taux plus élevés que dans l'Est du Canada. Les taux d'infections à Staphylococcus aureus résistant à la méthicilline (SARM) ont atteint un sommet en 2009 (9,5 infections à SARM pour 1000 hospitalisations), puis ont diminué en 2012 (8,8 infections pour 1000 hospitalisations), la région du Centre présentant des taux plus élevés que dans les régions de l'Ouest et de l'Est. Historiquement faibles, les taux d'entérocoques résistant à la vancomycine (ERV) ont augmenté à 0,08 infection pour 1000 hospitalisations en 2007, montant graduellement à 0,5 infection pour 1000 hospitalisations en 2012 (taux systématiquement plus élevés dans la région de l'Ouest, légèrement moins élevés dans la région du Centre et le plus faibles dans la région de l'Est). Les taux d'infections par les entérobactéries résistantes aux carbapénèmes (ERC), mesurés depuis 2010, sont faibles et stables (0,11 infection par ERC pour 1000 hospitalisations en 2010 et 0,14 infection pour 1000 hospitalisations en 2012), et sont plus élevés dans les régions de I'Ouest et du Centre comparativement à la région de l'Est.

Conclusion : Au Canada, parmi les quatre organismes résistants aux antibiotiques sous surveillance, les taux d'infections à Clostridium difficile associées aux soins de santé et à SARM ont graduellement diminué, tandis que ceux des infections à ERV sont faibles mais à la hausse, et ceux des infections à ERC demeurent faibles (taux systématiquement plus élevés dans les régions de l'Ouest et du Centre comparativement à la région de l'Est). 


\section{Introduction}

Même si l'émergence de souches résistantes de bactéries remonte aux débuts d'utilisation des antibiotiques dans les années 1940, la résistance aux antibiotiques est de plus en plus considérée comme un problème touchant les soins cliniques et la santé publique. La résistance aux antibiotiques s'est répandue dans le monde, affectant à la fois la santé humaine et l'approvisionnement alimentaire.

L'Agence de la santé publique du Canada (I'Agence ou l'ASPC) recueille des données au niveau national sur les organismes antimicrobiens résistants chez les humains par l'intermédiaire du Programme canadien de surveillance des infections nosocomiales (PCSIN). II s'agit d'un effort collaboratif réunissant le Centre de la lutte contre les maladies transmissibles et les infections, le Laboratoire national de microbiologie (LNM) et divers hôpitaux sentinelles de partout au pays participant comme membres du Comité canadien d'épidémiologistes hospitaliers (un sous-comité de l'Association pour la microbiologie médicale et l'infectiologie Canada ou AMMI). Le PCSIN vise à identifier les tendances et à orienter les programmes et politiques de prévention et de contrôle des infections partout au pays.

Les rapports de surveillance du PCSIN sont publiés sur une base régulière. L'objectif du présent article est de fournir un résumé des taux de résistance aux antibiotiques de quatre organismes clés résistants aux antibiotiques dans divers hôpitaux majeurs du Canada : Clostridium difficile (CD) (infection associée aux soins de santé), Staphylococcus aureus résistant à la méthicilline (SARM), entérocoques résistants à la vancomycine (ERV) et entérobactéries résistantes aux carbapénèmes (ERC). Le présent article examine les tendances chez les adultes et les enfants admis dans les hôpitaux participants du PCSIN, selon les hospitalisations par année. Le rapport le plus récent du PCSIN, intitulé Surveillance des microorganismes résistant aux antimicrobiens (MRA) - Rapport de surveillance d'après les données du

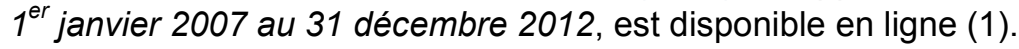

\section{Méthodologie}

\section{Collecte de données}

Les définitions de cas étaient préalablement convenues pour chacun des quatre organismes. Par suite de l'identification d'un cas dans l'un des hôpitaux participants, un questionnaire normalisé sur le patient, portant sur diverses informations démographiques et cliniques, notamment les hospitalisations antérieures au cours des 12 mois précédents et le siège de la culture positive, était rempli. Les données étaient ensuite soumises par voie électronique (par l'entremise d'un système de gestion de l'information sur le Web, le Réseau canadien de renseignements sur la santé publique [RCRSP]) à l'ASPC pour une analyse statistique plus approfondie et le stockage. Les hôpitaux participants devaient également fournir à l'Agence des données sur le nombre d'hospitalisations et le nombre de jours-patients durant l'année de surveillance correspondante (Remarque : Il existe de très légères différences entre les nombres rapportés dans le présent article et ceux du rapport en ligne, et ce, en raison de mises à jour récentes des centres participants; en cas de différence, il faut se fier aux données du présent article).

Les définitions normalisées de la surveillance du National Healthcare Safety Network (NHSN), qui comportent des critères tant cliniques que de laboratoire, ont servi à la confirmation des infections (en l'absence de signes cliniques, le patient était jugé comme étant colonisé plutôt qu'infecté).

\section{Analyse de laboratoire}

Conformément au protocole convenu pour chaque organisme, un échantillon d'isolats était envoyé au Laboratoire national de microbiologie (LNM) pour des tests moléculaires. Le LNM devait également envoyer les résultats de laboratoire à l'ASPC par l'entremise du RCRSP. Les résultats de laboratoire et les données recueillies au moyen des questionnaires liés au patient étaient associés à l'aide d'un identifiant patient anonyme unique. 


\section{Analyse des données}

Les données des hôpitaux participants et du LNM ont été extraites, validées et statistiquement analysées par le personnel de l'ASPC. Les taux d'infection par 1000 hospitalisations étaient calculés en divisant le nombre de cas par le nombre total d'hospitalisations par année multiplié par 1000 . Ils étaient calculés uniquement avec les données admissibles, c'est-à-dire celles (fournies par les hôpitaux) du numérateur (cas) et du dénominateur (hospitalisations). Pour les besoins du rapport et pour assurer la confidentialité des données, les provinces ont été regroupées en trois régions : Ouest (Colombie-Britannique, Alberta, Saskatchewan et Manitoba), Centre (Ontario et Québec) et Est (Nouvelle-Écosse, Nouveau-Brunswick, Île-du-Prince-Édouard et Terre-Neuve-et-Labrador) (I'Île-du-Prince-Édouard n'a commencé à présenter des données qu'en 2011; les territoires ne soumettent actuellement aucune donnée).

La surveillance dans les hôpitaux participants est considérée comme faisant partie du mandat des programmes de prévention et de contrôle des infections des hôpitaux et ne s'inscrit pas dans le cadre de la recherche sur des humains; par conséquent, aucune approbation auprès d'un comité d'examen éthique n'a été demandée.

\section{Résultats}

\section{Clostridium difficile associé aux soins de santé}

Clostridium difficile est la cause la plus répandue de diarrhée infectieuse et de colite pseudomembraneuse dans les hôpitaux et les établissements de soins de longue durée au Canada (2). C. difficile entraîne un éventail de réactions allant de l'absence de symptôme à une forme grave et potentiellement mortelle de la maladie (3). La population à risque de contracter une infection à Clostridium difficile inclut les personnes âgées, ainsi que les personnes qui prennent certains antibiotiques, qui sont immunodéprimées ou qui sont atteintes d'une grave maladie sous-jacente. Conformément à la définition de cas (voir l'encadré ci-dessous), seuls les cas de $C$. difficile associé aux soins de santé sont rapportés ici.

\section{Définition de cas d'infection à Clostridium difficile associée aux soins de santé}

Un patient est identifié comme présentant une infection à Clostridium difficile (ICD) :

- $\quad$ 'il présente une diarrhée ou de la fièvre, des douleurs abdominales et/ou un iléus, et si un résultat positif pour le $C$. difficile est confirmé en laboratoire par un essai de détection des toxines ou une réaction en chaîne de la polymérase (PCR);

- $\quad$ s'il a reçu un diagnostic de pseudomembranes à la sigmoïdoscopie ou à la colonoscopie ou un diagnostic histologique/pathologique d'ICD;

- si un diagnostic de syndrome colectasique a été posé (chez les patients adultes seulement).

En outre, un patient est considéré comme présentant une infection à Clostridium difficile associée aux soins de santé si :

- $\quad$ les symptômes de l'ICD apparaissent à l'hôpital moins de 72 heures après l'hospitalisation du patient;

- $\quad$ I'ICD survient chez un patient qui a déjà été hospitalisé et qui a obtenu son congé de cet hôpital au cours des quatre semaines précédentes.

Au Canada, les taux nationaux d'infections au Clostridium difficile (ICD) associées aux soins de santé ont atteint un sommet en 2008 (5,8 ICD associées aux soins de santé pour 1000 hospitalisations), puis sont demeurés stables entre 2009 et 2012 (environ 5 ICD associées aux soins de santé pour 1000 hospitalisations), les régions de l'Ouest et du Centre du Canada présentant des taux plus élevés que dans l'Est du Canada (Figure 1). 
FIGURE 1. Taux nationaux et régionaux d'incidence d'infection à Clostridium difficile (ICD) associée aux soins de santé pour 1000 hospitalisations, du 1er janvier 2007 au 31 décembre 2012 (N = 18871 )

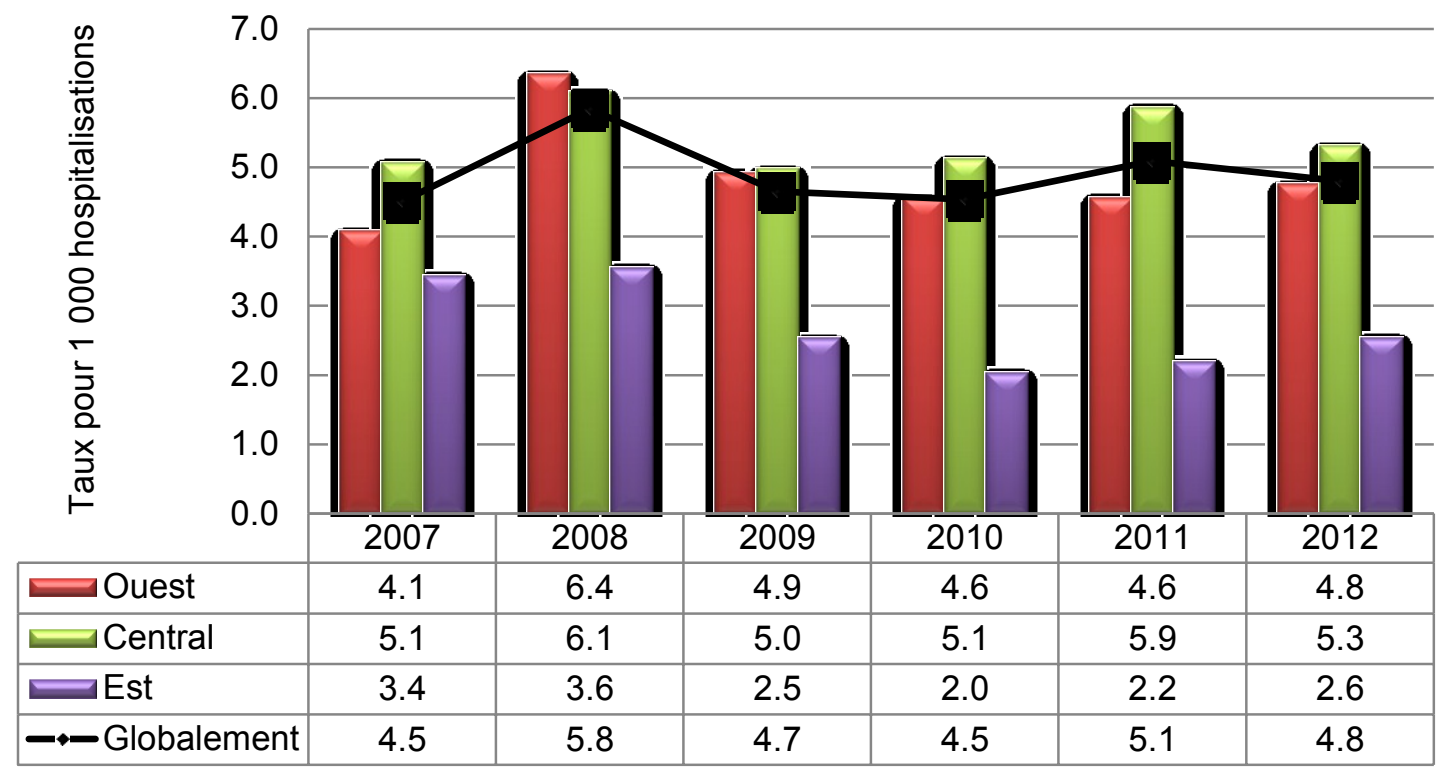

Dans l'ensemble, la souche de type 1 nord-américain en champ pulsé (NAP1) était la plus répandue. Parmi les 2497 échantillons de selles soumis, la souche NAP1 a été détectée dans $1059(42,4 \%)$ échantillons; la souche NAP4 dans $11,0 \%$ des échantillons et la souche NAP2 dans 10,3\% des échantillons (données non indiquées).

\section{Staphylococcus aureus résistant à la méthicilline (SARM)}

Staphylococcus aureus ( $S$. aureus) colonise habituellement la peau et les surfaces de muqueuses d'humains en bonne santé. Cette bactérie peut également causer des infections urinaires, de plaies ou de la peau et des tissus mous, une ostéomyélite, une endocardite et une bactériémie. La bactérie $S$. aureus peut être acquise dans la communauté, dans les hôpitaux ou dans d'autres milieux de soins de santé tels les établissements de soins de longue durée, les cliniques de dialyse ou les centres de réadaptation. $S$. aureus est connue pour développer facilement une résistance aux antibiotiques, ce qui explique en partie le lourd fardeau associé à la maladie dans le monde entier (4). SARM est un type de S. aureus devenue résistante aux bêta-lactamines (antibiotiques) administrées en première intention, telles la méthicilline, l'oxacilline, la pénicilline et l'amoxicilline. Conformément à la définition de cas (voir l'encadré ci-dessous), seuls les cas de Clostridium difficile associé aux soins de santé sont rapportés ici.

\section{Définition de cas d'infection à Staphylococcus aureus résistant à la méthicilline (SARM)}

- Isolement de Staphylococcus aureus, quel que soit le siège du prélèvement ET

- Résistance de l'isolat à l'oxacilline

ET

- Patient admis à l'hôpital

ET

- "Cas récemment identifié de SARM » dans un hôpital participant du PCSIN au moment de l'admission du patient à l'hôpital ou cas identifié pendant l'hospitalisation

Ceci comprend :

- Cas de SARM diagnostiqués pour la première fois pendant cette hospitalisation; 
- Cas déjà diagnostiqués dans un autre hôpital ne participant pas au PCSIN.

Les taux de SARM ont atteint un sommet en 2009 (9,5 infections pour 1000 hospitalisations), puis ont diminué en 2012 (8,8 infections pour 1000 hospitalisations). Les taux dans la région du Centre ont légèrement augmenté depuis 2008 et sont plus élevés que la moyenne nationale à chacune des années de surveillance (Figure 2).

FIGURE 2. Taux nationaux et régionaux d'infection et de colonisation par le Staphylococcus aureus résistant à la méthicilline (SARM) pour 1000 hospitalisations, du 1er janvier 2008 au 31 décembre 2012 ( $N=9650)$

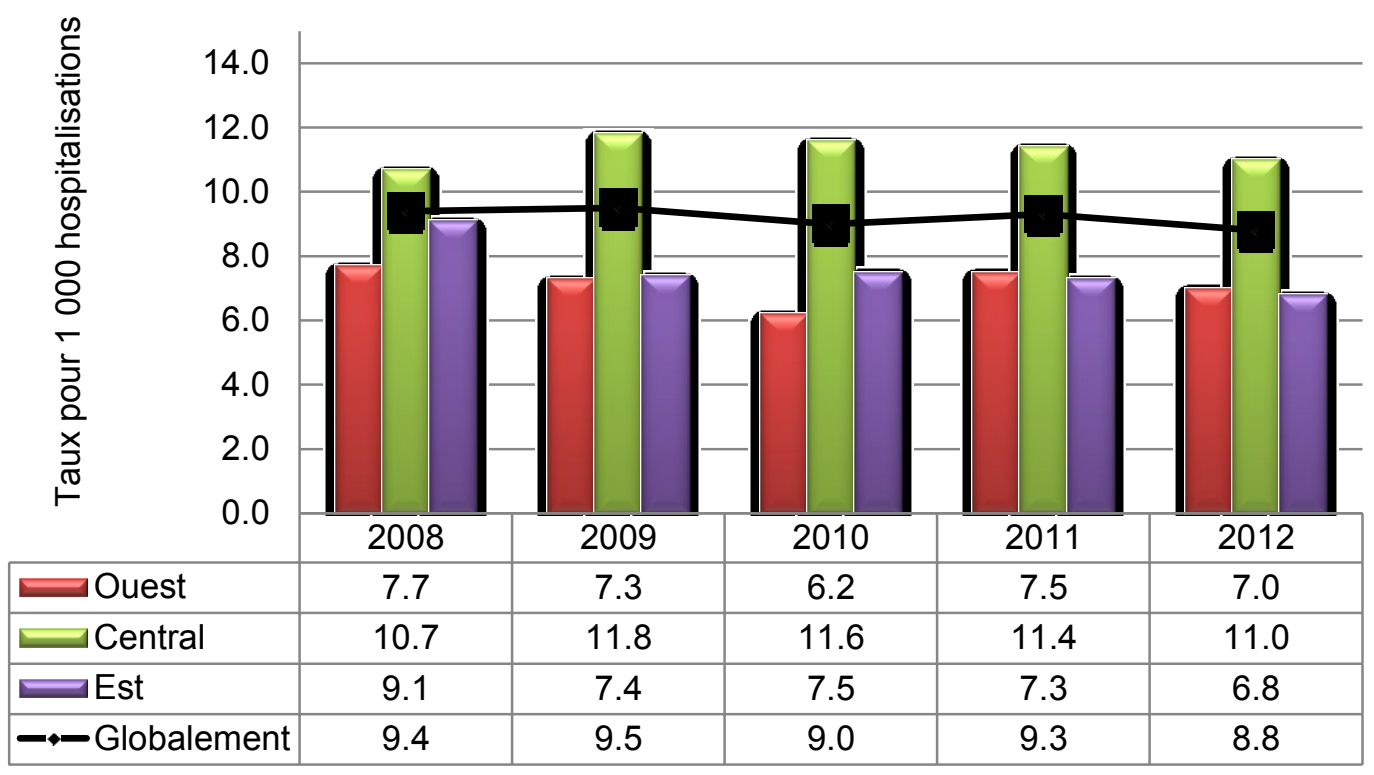

Les blessures de la peau et des tissus mous ou les brûlures étaient les sources les plus courantes des infections cliniques à SARM; ces dernières représentent, en moyenne, environ $40 \%$ de toutes les infections cliniques observées durant la période de surveillance. De 2008 à 2012, environ $9 \%$ des patients présentant une infection clinique par le SARM et $25 \%$ des patients présentant une bactériémie à SARM sont décédés 30 jours après la date de la culture positive.

\section{Entérocoques résistant à la vancomycine (ERV)}

Les infections à ERV touchent le plus souvent les personnes hospitalisées et immunodéprimées, les personnes ayant déjà reçu de la vancomycine ou d'autres antibiotiques de façon prolongée, les personnes vieillissantes et ayant subi des interventions chirurgicales, ainsi que les personnes possédant un dispositif médical à demeure tel qu'une sonde urinaire (voir l'encadré ci-dessous).

\section{Définition de cas d'entérocoques résistant à la vancomycine}

Tout patient hospitalisé chez qui la bactérie Enterococcus faecium ou Enterococcus faecalis présentant une concentration minimale inhibitrice de vancomycine $>8 \mu \mathrm{g} / \mathrm{mL}$ a été isolée dans un échantillon clinique.

Historiquement faibles, les taux d'ERV ont augmenté à 0,08 infection pour 1000 hospitalisations en 2007, montant graduellement à 0,5 infection pour 1000 hospitalisations en 2012 (taux systématiquement plus élevés dans la région de l'Ouest, légèrement moins élevés dans la région du Centre et le plus faibles 
dans la région de l'Est) (Figure 3). Le gène vanA demeure prédominant parmi les bactériémies associées aux ERV.

FIGURE 3. Taux nationaux et régionaux d'incidence d'infections d'entérocoques résistant à la vancomycine (ERV) pour 1000 hospitalisations, du 1er janvier 2007 au 31 décembre 2011 ( $N=1510$ )

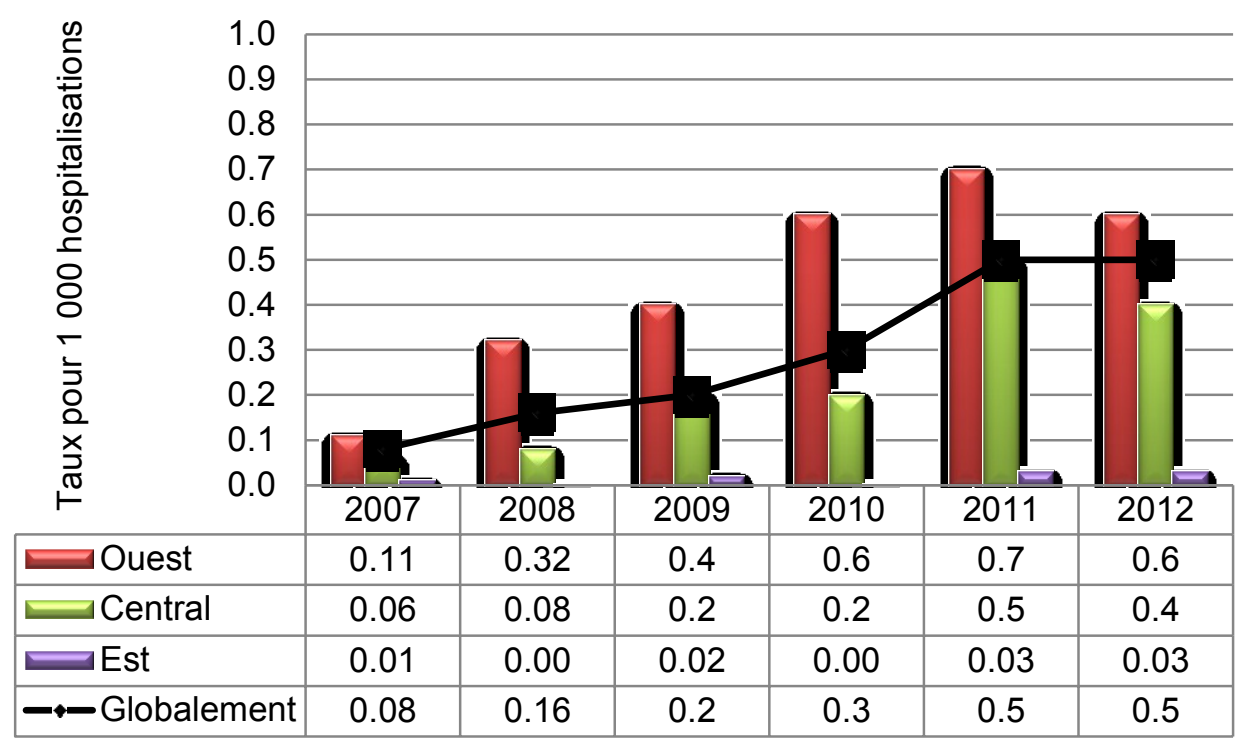

\section{Entérobactéries résistantes aux carbapénèmes (ERC)}

Les bacilles Gram négatif causent toute une variété de maladies, allant de la pneumonie aux infections urinaires, et des infections de plaies aux septicémies. Elles se produisent habituellement chez des patients malades exposés à des milieux de soins de courte ou de longue durée. Les infections sont habituellement causées par des espèces appartenant à la famille des entérobactéries, comme Escherichia coli, Klebsiella pneumoniæ et Enterobacter cloaca. Ces bactéries sont souvent résistantes à de nombreux antibiotiques couramment prescrits, mais elles demeurent habituellement susceptibles au groupe des carbapénèmes. II n'existe aucun nouvel antibiotique en cours de développement contre les bactéries résistantes aux carbapénèmes. La définition de cas d'infection par une entérobactérie résistante aux carbapénèmes a évolué au fil du temps (voir l'encadré ci-dessous).

\section{Définition de cas d'isolats d'entérobactéries résistantes aux carbapénèmes}

Du $1^{\text {er }}$ janvier 2010 au 31 août 2010, tout patient porteur d'une entérobactérie dont la concentration minimale inhibitrice $(\mathrm{CMI})$ d'un des trois carbapénèmes (imipénème, méropénème, értapénème) était $\geq 2 \mu \mathrm{g} / \mathrm{ml}$ ou dont le diamètre de diffusion avec disque était $\leq 21 \mathrm{~mm}$ était admissible à l'inclusion.

Du $1^{\text {er }}$ septembre 2010 au 31 décembre 2012, la CMI pour l'ertapénème a été réduite à $\geq 0,5 \mu \mathrm{g} / \mathrm{ml}$ et un diamètre de diffusion avec disque pour tout carbapénème $\leq 22 \mathrm{~mm}$ a été jugé admissible à l'inclusion.

Les taux d'infections par les entérobactéries résistantes aux carbapénèmes (ERC), mesurés depuis 2010 , sont faibles et stables $(0,11$ infection pour 1000 hospitalisations en 2010 et 0,14 infection pour 1000 hospitalisations en 2012), et sont plus élevés dans les régions de l'Ouest et du Centre comparativement à la région de l'Est. 
FIGURE 4. Taux nationaux et régionaux d'infections d'entérobactéries résistantes aux carbapénèmes (ERC) pour 1000 hospitalisations, du 1er janvier 2010 au 31 décembre 2012 ( $N=231$ )

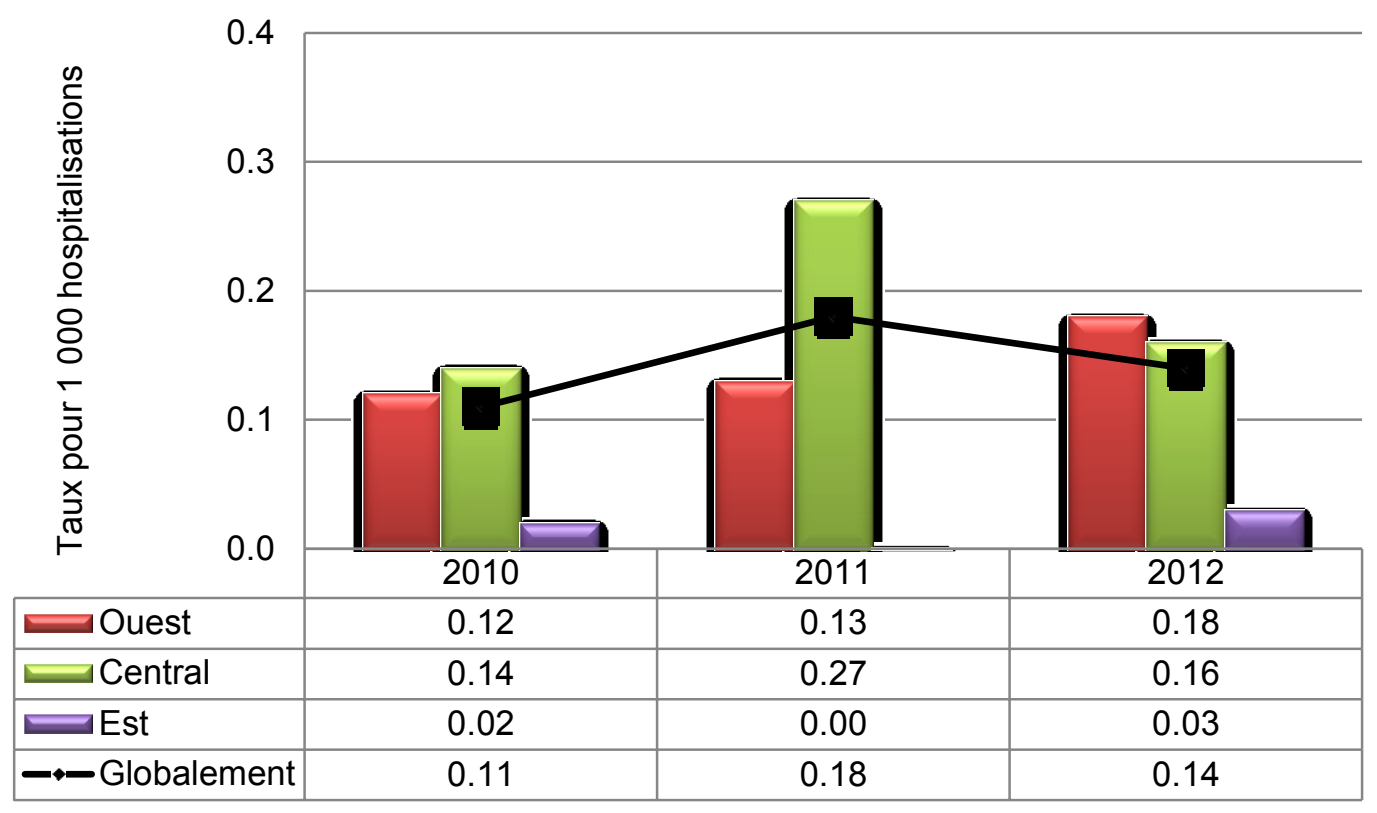

\section{Discussion}

Au Canada, au cours des dernières années, parmi les quatre organismes résistants aux antibiotiques sous surveillance, les infections à Clostridium difficile associées aux soins de santé et les infections à Staphylococcus aureus résistant à la méthicilline ont graduellement diminué, tandis que celles aux entérocoques résistant à la vancomycine ont été peu nombreuses (mais sont actuellement à la hausse). Les infections aux entérobactéries résistantes aux carbapénèmes demeurent quant à elles peu nombreuses (taux systématiquement plus élevés dans les régions de l'Ouest et du Centre comparativement à l'Est du Canada).

Il est difficile d'établir des comparaisons entre les taux canadiens et les taux internationaux en raison de différences au niveau des définitions et des analyses. Par exemple, certains pays ne font état que des bactériémies à SARM plutôt que tous les types d'infections à SARM, alors que d'autres pays rapportent leurs taux par 100000 habitants (au Canada, les taux sont calculés par hospitalisations). En outre, les taux observés dans divers pays et régions du monde montrent des variations régionales. Toutefois, les tendances générales au Canada ne sont pas différentes de celles observées ailleurs dans le monde.

Plusieurs limites doivent être prises en compte dans le cadre de l'interprétation des données présentées dans le présent article. Premièrement, les données de surveillance du PCSIN sous-estiment probablement l'ampleur du phénomène, car elles ne reflètent pas le nombre total de patients hospitalisés au Canada atteints de ces infections. Ces données ne peuvent nous renseigner que sur les patients hospitalisés qui ont été testés et diagnostiqués avec ce type d'infection, et non sur ceux qui n'ont pas encore été testés et diagnostiqués. Les cas observés en consultation externe, comme dans les services d'urgence et les cliniques, ne sont pas pris en compte par ce système de surveillance. Les hôpitaux participants (souvent de grands centres de soins tertiaires de courte durée situés dans les grandes villes) ne sont pas nécessairement représentatifs de tous les hôpitaux canadiens. Les pratiques de prescription d'antibiotiques et la mise en œuvre des mesures de prévention et de contrôle des infections peuvent varier d'un hôpital à l'autre; cependant, comme ce système de surveillance ne recueille pas de données sur ces facteurs, il n'est pas possible d'établir une corrélation entre ces facteurs et ces infections. Comme à l'habitude, ces résultats sont sujets à changement alors que de nouvelles données sont mises à disposition. 
La résistance aux antimicrobiens continuera à mettre en danger la santé des Canadiens et des autres habitants de la planète pendant encore un certain temps. La surveillance aidera à documenter les progrès que nous continuerons de faire pour freiner cette résistance.

\section{Remerciements}

Les auteurs tiennent à remercier toutes les personnes impliquées dans le PCSIN (dans les hôpitaux participants) de leur avoir fourni les données confidentielles non nominatives. Sans leur étroite collaboration et leur participation, l'analyse et la présentation de ces données n'auraient pas été possibles. La liste de tous les collaborateurs se trouve dans le rapport complet (1).

\section{Conflit d'intérêts}

II n'y a aucun conflit d'intérêts à déclarer.

\section{Financement}

Ce travail a été appuyé par l'Agence de la santé publique du Canada.

\section{Références}

(1) Agence de la santé publique du Canada (ASPC). Surveillance des micro-organismes résistant aux antimicrobiens : Rapport de surveillance sur résultats du $1^{\mathrm{er}}$ janvier 2007 au 31 décembre 2012. Ottawa : Programme canadien de surveillance des infections nosocomiales, ASPC; mis à jour en février 2014. http://www.phac-aspc.gc.ca/amr-ram/assets/pdf/aro-mra-2007-2012-fra.pdf

(2) Pouteen SM, Simor AE. Clostridium difficile-associated diarrhea in adults. CMAJ. 2004; 171(1):51-8.

(3) Bartlett JG. Antibiotic-associated diarrhea. New Engl J Med. 2002; 346:334-9.

(4) Otto, M. Basis of virulence in community-associated methicillin-resistant staphylococcus aureus. Annual Review of Microbiology. 2010; 64:143-62. 